\title{
Self-Repelling Snakes for Topology-Preserving Segmentation Models
}

\author{
Carole Le Guyader and Luminita A. Vese, Member, IEEE
}

\begin{abstract}
The implicit framework of the level-set method has several advantages when tracking propagating fronts. Indeed, the evolving contour is embedded in a higher dimensional level-set function and its evolution can be phrased in terms of a Eulerian formulation. The ability of this intrinsic method to handle topological changes (merging and breaking) makes it useful in a wide range of applications (fluid mechanics, computer vision) and particularly in image segmentation, the main subject of this paper. Nevertheless, in some applications, this topological flexibility turns out to be undesirable: for instance, when the shape to be detected has a known topology, or when the resulting shape must be homeomorphic to the initial one. The necessity of designing topology-preserving processes arises in medical imaging, for example, in the human cortex reconstruction. It is known that the human cortex has a spherical topology so throughout the reconstruction process this topological feature must be preserved. Therefore, we propose in this paper a segmentation model based on an implicit level-set formulation and on the geodesic active contours, in which a topological constraint is enforced.
\end{abstract}

Index Terms-Additive operator splitting (AOS) scheme, geodesic active contours, level-set method, segmentation, topology preservation.

\section{INTRODUCTION}

$\mathbf{S}$ EGMENTATION processing mainly consists of detecting and visualizing the boundaries of the objects contained in a given image. One of the well-known tools to perform this partition of the image is the active contour model (also called snake model, or deformable model), as proposed in the work by Kass et al.[17]. Starting with a contour that encloses the object to be detected, deformable models aim at making this contour evolve so that it matches the edge of the considered object.

The shape taken by the contour throughout the process is related to an energy-minimization problem. The energy functional that is minimized is a weighted combination of internal forces (describing properties of elasticity and rigidity of the contour)

Manuscript received March 12, 2007; revised December 27, 2007. This work was supported in part by an Alfred P. Sloan fellowship, in part by the National Institutes of Health (NIH) through the NIH Roadmap for Medical Research Grant U54 RR021813 entitled Center for Computational Biology (CCB), and in part by the National Science Foundation Grant DMS 0312222. Part of this work was done while C. Le Guyader was visiting the Department of Mathematics, UCLA. The associate editor coordinating the review of this manuscript and approving it for publication was Dr. Gaudenz Danuser.

C. Le Guyader is with IRMAR, UMR CNRS 6625, Institut National des Sciences Appliquées de Rennes, 35043 Rennes Cedex, France (e-mail: cleguyad@insa-rennes.fr).

L. A. Vese is with the Department of Mathematics, University of California, Los Angeles, CA 90095-1555 USA (e-mail: ivese@math.ucla.edu).

Color versions of one or more of the figures in this paper are available online at http://ieeexplore.ieee.org.

Digital Object Identifier 10.1109/TIP.2008.919951 and external forces (that guide the curve towards the boundary of the object).

In this parametric model [17], the introduced functional is nonintrinsic since it depends on the parameterization and is not related to the geometry of the object to be detected. Besides, in this framework, topology is maintained by the Lagrangian formulation.

Extensive work has been done in a similar way by other contributors, such as Cohen et al. [9], Cohen et al. [8], or McInerney et al. [20], to improve the overall accuracy of the modeling.

In Caselles et al. [5] and Kichenassamy et al. [29], the authors focus on a particular active contour model (called "geodesic active contours") and prove that it is equivalent to finding a geodesic curve in a Riemann space whose metrics is linked to the image content.

To establish the evolution equation of the contour, a family of curves depending on a parameter $t \geq 0$ representing time is defined. The derivative of the functional with respect to $t$ is computed and one seeks the direction for which the energy decreases the most rapidly.

The level-set formulation can be straightforwardly derived using the theory of curve evolution (see [21], [23], and [34], for instance). The implicit representation of the curve via a level-set function avoids parameterization issues. The discretization of the problem is made on a fixed rectangular grid. The model is intrinsic (if we define a new parameterization, the energy is unchanged), allows for corners, and the initial condition does not necessarily have the same topology as the resulting contour. The geometric properties of the contour (curvature, unit normal vector) are easily deduced from the level-set function.

The topological flexibility is a great benefit since it allows the simultaneous detection of several objects in the image, which was not possible in the case of parametric deformable models without additional modifications or additional work. However, in some applications, this flexibility is undesirable. For instance, when the final shape must be homeomorphic to the initial one, or when the topology of the final contour must be consistent with the known topology of the object. In brain imaging, for example, when located near a lobe, parts of the contour may fold up and finally have a contact point (without merging), which is not consistent with the spherical topology of the brain. The contrast of the image can also be impaired and the number of components of the object can thus be increased.

These remarks motivated the work presented next: to keep the benefits of the level-set framework (parameter-free, intrinsic, easily deduced geometrical properties of the front, etc.), we propose a new nonparametric topology-constrained segmentation model based on the geodesic active contours [5], [29]. 


\section{A. Prior Related Work}

1) Work of Han et al.: Our work is much motivated by the works [14]-[16], in which Han et al. propose a first approach to preserve the topology of the implicit contour while the embedding level-set function is evolving. The key idea of the model lies in the concept of simple point from digital topology. The authors assume that the topology of the zero level set is equivalent to the topology of the boundary of the digital object it defines. The topology-preservation problem is, therefore, simplified in the following way. The topology of the implicit contour can change only if the level-set function changes sign at a grid point. This is only a necessary condition: not every change of sign of the level-set function implies a topology change of the zero level set and consequently of the digital object boundary. One needs to introduce the concept of simple point to identify grid points for which, when removed or added, topology is no longer preserved: a grid point is said to be simple if it can be added or removed without changing the topology of the object. The computation of two topological numbers is sufficient to characterize simple points. Thus, Han et al. introduce an algorithm that at each iteration monitors the changes of sign of the level-set function and prevents the level-set function from changing sign on grid points which are not simple. The procedure is, therefore, pixel based. The authors also stress the arbitrariness of the result produced by the algorithm, depending on the order in which points are treated in the narrow band. Their method has been applied to the geodesic active contour model [5]. Here, we propose to define, in the continuum case, a geodesic-active-contour-based segmentation model that globally integrates a topological constraint emerging from a geometrical remark.

2) Work of Alexandrov and Santosa: Our work is also much motivated by the interesting work [1], which is a curve evolution method based on level sets for shape optimization problems arising in material science. The signed-distance function is used, and their proposed method avoids overlaps of the narrow band of the evolving contour. Their method, like ours, is a variational one, but it has not been proposed for image segmentation. Only artificial tests of shape optimization illustrate their point in [1]. In the case of a shape optimization problem, their model minimizes $F_{\mu}(\Phi)=F(\Phi)+\mu H(\Phi)$, with $\mu \ll 1$, where $F(\Phi)$ is a general shape optimization functional and $H(\Phi)$ is the topology constraint term defined by

$$
\begin{aligned}
H(\Phi)=-\int_{\partial D} \log [\phi(x+d \nabla \phi(x))] d s & \\
& -\int_{\partial D} \log [-\phi(x-l \nabla \phi(x))] d s
\end{aligned}
$$

where $D=\{x: \Phi(x)>0\}$ and $d>0, l>0$ are given parameters. The unknown level-set function $\Phi$ must be a signeddistance function.

3) Work of Sundaramoorthi and Yezzi: Another related and very interesting approach based on the knot energy is presented in [36] and [37]. This also includes a penalty in the segmentation functional in the form of a double integral that could prevent a curve $C$ from changing topology. The relevant term that is minimized inspired from the knot energy is

$$
E(C)=\frac{1}{2} \iint_{C \times C} \frac{d p d p^{\prime}}{\left\|C(p)-C\left(p^{\prime}\right)\right\|^{\gamma}}
$$

where $\|\cdot\|$ is the usual Euclidean norm and $\gamma>0$. The topology depends on the choice of the parameter $\gamma$. This term is also coupled with the method from [15] and [16] to insure that the topology is always preserved. Experimental results similar to ours are presented in [36] and [37].

4) Work of Rochery et al.: This related work [25]-[27], although devoted to a different problem, uses a similar idea to ours to avoid that pieces of the same curve come in contact to merge or break. The goal is to track thin long objects that evolve, with applications to the automatic extraction of road networks in remote sensing images. In [25]-[27], the authors have proposed interesting non local regularization terms on a curve $C$ parameterized by $p \in[0,1]$. In [25], these are of the form $E(C)=-\int_{0}^{1} \int_{0}^{1} \vec{t}(p) \cdot \vec{t}\left(p^{\prime}\right) \Psi\left(\left\|C(p)-C\left(p^{\prime}\right)\right\|\right) d p d p^{\prime}$, where $\vec{t}(p), \vec{t}\left(p^{\prime}\right)$ are the tangent vectors to the curve at points $C(p), C\left(p^{\prime}\right)$, and $\left\|C(p)-C\left(p^{\prime}\right)\right\|$ is again the Euclidean distance between the curve points $C(p)$ and $C\left(p^{\prime}\right)$. The function $\Psi$ is chosen to be $\Psi(l)=\sinh ^{-1}(1 / l)+l-\sqrt{1+l^{2}}$, thus decreasing on $[0, \infty[$. Other nonlocal forms are considered as well, and geometric motions of thin long objects are obtained. As in our case, the implicit representation by level sets is used for implementation in [25] and [27]. In [26], the authors carry on their ideas, but this time in a phase-field approach for the representation of the motion. The work [25] is continued with more details in [27]. However, although related, our proposed approach is different in several aspects, as will be seen later.

5) Work of Cecil et al.: In a preliminary work, the Ph.D. dissertation of Cecil [6] (in collaboration with Osher and the second author), is also devoted to this problem, with satisfactory partial results based on the active contour model without edges [7]. The penalty is defined in a completely different way, but for more details we refer the reader to [6].

Finally, for completeness, we also refer the reader to other segmentation methods using variational approaches and active contours using coupled curve evolution equations, even if these are not directly related to the main ingredients of the proposed approach. These include the work of Zhao et al. [43], Samson et al. [30], [31], Chan and the second author [40], Paragios and Deriche [24], Yezzi et al. [39], [42], and Dufour et al. [13], among others.

The outline of the paper is as follows. Section II is devoted to the depiction of the model. Section III presents the associated evolution problem (with its derivation details given in Appendix A, and its numerical discretization in Appendix B). We conclude the paper with experimental results on various synthetic and real images, in 2-D or 3-D, in Section IV.

\section{DEPICTION OF THE MODEL}

Let $\Omega$ be a bounded open subset of $\mathbb{R}^{n}, \partial \Omega$ its boundary and let $I$ be a given bounded image function defined by $I: \bar{\Omega} \rightarrow \mathbb{R}$. For the purpose of illustration, we consider $n=2$.

1) Review of the Geodesic Active Contour Model: We first recall one of the main ingredients of the proposed model, the geodesic active contour model [5], [29] for boundary detection using the gradient of the image data as an edge detector. Let us denote by $C$ the set of closed parameterized curves such that $C=\left\{\mathcal{C}:[0,1] \rightarrow \mathbb{R}^{2}\right.$ piecewise $\left.\mathcal{C}^{1}, \mathcal{C}(0)=\mathcal{C}(1)\right\}$ (these 
curves are parameterized by $q \in[0,1])$. In [5] and [29], the authors introduce the following minimization problem:

$$
\min _{\mathcal{C} \in C}\left\{J(\mathcal{C})=\int_{0}^{1} g(|\nabla I(\mathcal{C}(q))|)\left|\mathcal{C}^{\prime}(q)\right| d q\right\}
$$

where $g:[0,+\infty[\rightarrow[0,+\infty[$ is an edge-detector function satisfying $g(0)=1, g$ strictly decreasing and $\lim _{r \rightarrow+\infty} g(r)=0$. This can be minimized using a gradient descent method by constructing a family of curves $\mathcal{C}(t, \cdot)$ for $t \geq 0$, such that $\mathcal{C}(0, \cdot)$ is an initial curve, and as $t$ increases, $J(\mathcal{C}(t, \cdot))$ decreases in time towards a local minimizer.

The modeling is then cast in the level-set setting [11], [12], [23]. The evolving contour is embedded in a higher dimensional Lipschitz continuous function $\Phi$ defined by $\Phi: \Omega \times[0,+\infty[\rightarrow$ $\mathbb{R}$, with $(x, t) \mapsto \Phi(x, t)$, such that $\mathcal{C}(t, \cdot)=\{x \in \Omega \mid \Phi(x, t)=$ $0\}$ and

$$
\left\{\begin{array}{l}
\Phi<0 \text { on } \omega \text { the interior of } \mathcal{C} \\
\Phi>0 \text { on } \Omega \backslash \bar{\omega}
\end{array}\right.
$$

The function $\Phi$ is preferred to be a signed-distance function for the stability of numerical computations (over time, one may periodically reinitialize the level-set function $\Phi$ as the signeddistance function to the set $\{x \in \Omega \mid \Phi(x)=0\}$; see [28] and [38], for instance).

Denoting by $\delta$ the 1-D Dirac measure, the length of the zero level set is given by [43]: $\int_{\Omega} \delta(\Phi(x))|\nabla \Phi(x)| d x$. We could have straightforwardly phrased the segmentation problem (1) in the level-set framework by minimizing the functional $F$ defined by

$$
F(\Phi)=\int_{\Omega} g(|\nabla I(x)|) \delta(\Phi(x))|\nabla \Phi(x)| d x
$$

Computing the Euler-Lagrange equation and using a gradient-descent method (similar to [7] and [43]), we would have then obtained the following evolution equation:

$$
\frac{\partial \Phi}{\partial t}=\delta_{\epsilon}(\Phi) \operatorname{div}\left(g(|\nabla I|) \frac{\nabla \Phi}{|\nabla \Phi|}\right)
$$

$\delta_{\epsilon}$ being a $\mathcal{C}^{1}$ regularization of the Dirac measure. As in [43], the scale factor $\delta_{\epsilon}(\Phi)$ is replaced by $|\nabla \Phi|$, to extend the motion uniformly to all level sets of $\Phi$.

2) Description of the Proposed Topology-Constrained Problem: The segmentation method we propose is based on the geodesic active contour model and includes a topological

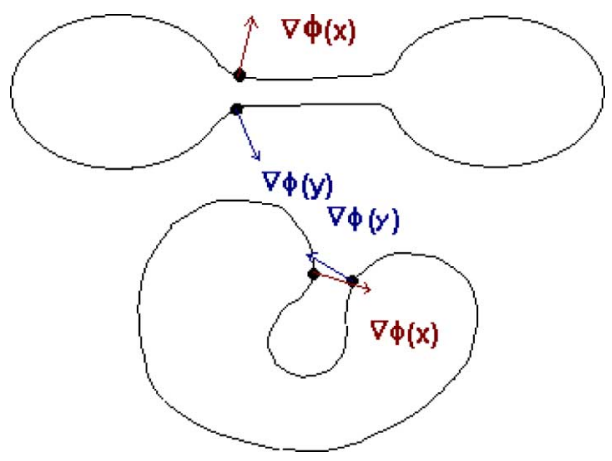

Fig. 1. Geometrical characterization of points in a zone where the curve is to split, merge, or have a contact point.

constraint based on a geometrical observation. In the following, we assume that $\Phi$ is a signed-distance function to $\mathcal{C}$ :

$$
\phi(x)= \begin{cases}\operatorname{dist}(x, \mathcal{C}), & \text { if } x \in \Omega-\bar{\omega} \\ -\operatorname{dist}(x, \mathcal{C}), & \text { if } x \in \omega .\end{cases}
$$

In that case, $\Phi$ satisfies $|\nabla \Phi|=1$. The gradient $\nabla \Phi$ is perpendicular to the isocontours of $\Phi$. It points in the direction of increasing $\Phi$, so the unit outward normal vector to the zero level line at point $x$ is merely $\nabla \Phi(x)$ (more generally, the unit outward normal vector to the $k$ level line at point $x$ is $\nabla \Phi(x)$ ). Also, $l$ denoting a real number, the set $\{x \in \Omega \mid \Phi(x)=l\}$ is obtained by shifting the points from the zero level line $\mathcal{C}$ by a quantity $l$ in the outward normal to $\mathcal{C}$, that is

$$
\{x \in \Omega \mid \Phi(x)=l\}=\{x+l \nabla \Phi(x), x \in \mathcal{C}\} .
$$

Let us now consider two points $(x, y) \in \Omega \times \Omega$ belonging to the zero level line of $\Phi$, close enough to each other, and let $\nabla \Phi(x)$ and $\nabla \Phi(y)$ be the unit outward normal vectors to the contour at these points. As shown in Fig. 1, when the contour is about to merge, split or have a contact point (that is when the topology of the evolving contour is to change), then $\langle\nabla \Phi(x), \nabla \Phi(y)\rangle$ $\simeq-1,\langle\cdot, \cdot\rangle$ denoting the Euclidean scalar product in $\mathbb{R}^{2}$.

Instead of working with only the points of the zero level line, we propose to focus on the points contained in a narrow band around the zero level line, more precisely, on the set of points $\{x \in \Omega \mid-l \leq \Phi(x) \leq l\}, l$ being a level parameter. We plan to minimize the following functional:

$$
F(\Phi)+\mu E(\Phi)
$$

$F$ being the functional of the geodesic active contour model (2) and $E$ the functional defined by (6), shown at the bottom of the page. The potential $G$ such that $G\left(\|x-y\|_{2}^{2}\right)=$

$$
\begin{aligned}
& E(\Phi)=-\int_{\Omega} \int_{\Omega}\left[\exp \left(-\frac{\|x-y\|_{2}^{2}}{d^{2}}\right)\right. \\
& \cdot\langle\nabla \Phi(x), \nabla \Phi(y)\rangle H(\Phi(x)+l) H(l-\Phi(x)) \\
& \text { - } H(\Phi(y)+l) H(l-\Phi(y))] d x d y \\
& =\int_{\Omega} \int_{\Omega}\left[G\left(\|x-y\|_{2}^{2}\right) g(\nabla \Phi(x), \nabla \Phi(y)) h(\Phi(x)) h(\Phi(y))\right] d x d y
\end{aligned}
$$


$\exp \left(-\|x-y\|_{2}^{2} / d^{2}\right)$ measures the nearness of the two points $x$ and $y$. Here, $H$ denotes the 1-D Heaviside function, and, therefore, $h(\Phi(x))=H(\Phi(x)+l) H(l-\Phi(x))=$ $\chi_{\{x \in \Omega,-l \leq \Phi(x) \leq l\}}(x)$. In the calculations, the Heaviside function being not differentiable, it is replaced by a smooth approximation denoted by $H_{\epsilon}$. At last, setting $g(\nabla \Phi(x), \nabla \Phi(y))=-\langle\nabla \Phi(x), \nabla \Phi(y)\rangle$, if the unit outward normal vectors to the level lines passing through $x$ and $y$ have opposite directions, then the functional is not minimal. In the following, we will use the notations $g=g\left(z_{1}, z_{2}, z_{3}, z_{4}\right)$ and we will denote by $\nabla_{\left(z_{1}, z_{2}\right)} g=\left(g_{z_{1}}, g_{z_{2}}\right)^{T}$ and by $\nabla_{\left(z_{3}, z_{4}\right)} g=\left(g_{z_{3}}, g_{z_{4}}\right)^{T}$. Here, $\mu>0$ is a tuning parameter [note that we use the same notation $g(|\nabla I|)$ for the edge function in (1), and also for the function $g\left(z_{1}, z_{2}, z_{3}, z_{4}\right)$ in (6), but we think that there will be no confusion].

\section{EVOLUTION PROBLEM}

We give in Appendix A the details of the derivation of the Euler-Lagrange equation associated with the minimization problem $\min _{\Phi} F(\Phi)+\mu E(\Phi), \mu>0$. Parameterizing the gradient descent direction by an artificial time $t \geq 0$, the Euler-Lagrange equation in $\Phi(x, t)$ with $\Phi(x, 0)=\Phi_{0}(x)$ (signed-distance function to the initial contour) and $x=\left(x_{1}, x_{2}\right) \in \Omega$, $y=\left(y_{1}, y_{2}\right) \in \Omega$ is

$$
\begin{aligned}
\frac{\partial \Phi}{\partial t}= & \delta_{\epsilon}(\Phi) \operatorname{div}\left(g(|\nabla I|) \frac{\nabla \Phi}{|\nabla \Phi|}\right) \\
+ & 4 \frac{\mu}{d^{2}} H_{\epsilon}(\Phi(x)+l) H_{\epsilon}(l-\Phi(x)) \\
& \cdot \int_{\Omega}\left[\left(\left(x_{1}-y_{1}\right) \frac{\partial \Phi}{\partial y_{1}}(y)+\left(x_{2}-y_{2}\right) \frac{\partial \Phi}{\partial y_{2}}(y)\right)\right. \\
& \left.\cdot e^{-\|x-y\|_{2}^{2} / d^{2}} H_{\epsilon}(\Phi(y)+l) H_{\epsilon}(l-\Phi(y))\right] d y .
\end{aligned}
$$

We replace $\delta_{\epsilon}(\Phi)$ by $|\nabla \Phi|$ (rescaling stage, see Zhao et al. [44] and Alvarez et al. [2]) and consider Neumann homogeneous boundary conditions: $\partial \Phi / \partial \vec{\nu}=0$ on $\partial \Omega$ with $\vec{\nu}$ denoting the unit outward normal to the boundary of $\Omega$. The speed of convergence can be increased by adding the component $k g(|\nabla I|)|\nabla \Phi|$ in the evolution equation, $k$ being a constant. Thus, we consider the following evolution problem, shown at the bottom of the page.

Details of the numerical implementation are given in Appendix B.

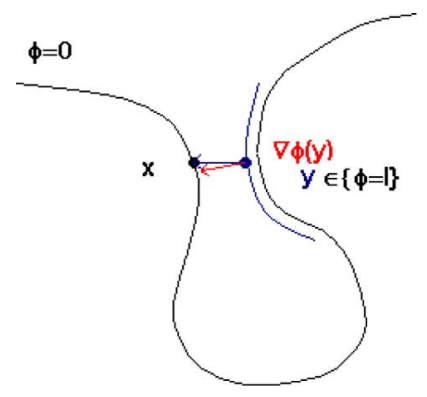

Fig. 2. Case where $x$ belongs to $\mathcal{C}$ and y belongs to the $l$ level line, $x$ and $y$ close enough.

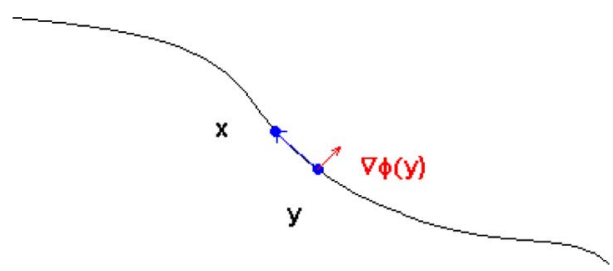

Fig. 3. Case where the two points are close enough and both belong to $\mathcal{C}$.

Remark: Let us consider the zero level line $\mathcal{C}$. A calculation gives that $\mathcal{C}$ moves according to

$$
\begin{array}{r}
\frac{\partial \mathcal{C}}{\partial t}=g(|\nabla I|) \kappa \vec{n}-\langle\nabla g(|\nabla I|), \vec{n}\rangle \vec{n}+k g(|\nabla I|) \vec{n} \\
+\frac{\mu^{\prime}}{|\nabla \Phi|}\left[\int _ { \Omega } \left(\langle x-y, \nabla \Phi(y)\rangle e^{-\|x-y\|_{2}^{2} / d^{2}}\right.\right. \\
\left.\left.\cdot H_{\epsilon}(\Phi(y)+l) H_{\epsilon}(l-\Phi(y))\right) d y\right] \vec{n}
\end{array}
$$

$\vec{n}$ being the unit inward normal vector, $\kappa$ the curvature and $\mu^{\prime}>$ 0 a constant. Consider now two points $x$ and $y$ close enough to each other, $x$ belonging to $\mathcal{C}$ and $y$ belonging to the narrow band of width $2 l$. In Fig. 2, the contour $\mathcal{C}$ is about to merge or to have a contact point. If we compute $\langle x-y, \nabla \Phi(y)\rangle$, we get a positive quantity.

If we now take two points $x$ and $y$ close enough to each other that both belong to $\mathcal{C}$ (Fig. 3), the contribution $\langle x-y, \nabla \Phi(y)\rangle$ is almost zero. Last, in the case of Fig. $4,\left\langle x-y_{1}, \nabla \Phi\left(y_{1}\right)\right\rangle$ is positive while $\left\langle x-y_{2}, \nabla \Phi\left(y_{2}\right)\right\rangle$ is negative. Globally, the contribution is zero in this part of the narrow band. Thus, in critical cases (when the curve is about to change topology), we can expect to have a positive quantity of the component $\int_{\Omega}\langle x-$ $y, \nabla \Phi(y)\rangle e^{-\|x-y\|_{2}^{2} / d^{2}} H_{\epsilon}(\Phi(y)+l) H_{\epsilon}(l-\Phi(y)) d y$, and then

$$
\left\{\begin{aligned}
\frac{\partial \Phi}{\partial t}= & |\nabla \Phi|\left[\operatorname{div}\left(g(|\nabla I|) \frac{\nabla \Phi}{|\nabla \Phi|}\right)+k g(|\nabla I|)\right]+4 \frac{\mu}{d^{2}} H_{\epsilon}(\Phi(x)+l) H_{\epsilon}(l-\Phi(x)) \\
& \cdot \int_{\Omega}\left[\left(\left(x_{1}-y_{1}\right) \frac{\partial \Phi}{\partial y_{1}}(y)+\left(x_{2}-y_{2}\right) \frac{\partial \Phi}{\partial y_{2}}(y)\right) e^{-\|x-y\|_{2}^{2} / d^{2}} H_{\epsilon}(\Phi(y)+l) H_{\epsilon}(l-\Phi(y))\right] d y \\
\Phi(x, 0)= & \Phi_{0}(x) \\
\frac{\partial \Phi}{\partial \vec{\nu}}= & 0, \text { on } \partial \Omega
\end{aligned}\right.
$$




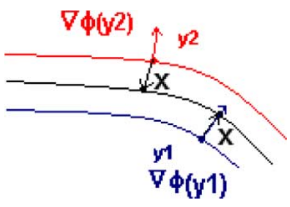

Fig. 4. Case where the two points are close enough, $x$ belonging to $\mathcal{C}$ and $y$ belonging to the $l$ level line or $-l$ level line.

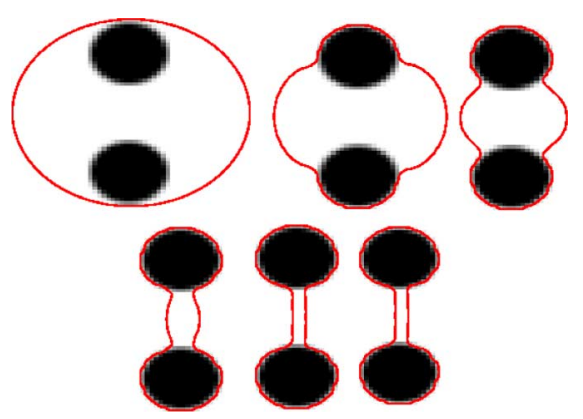

Fig. 5. Segmentation of the synthetic image with two disks when topological constraints are applied. The parameters are: $h=1, \tau=0.5, l=1$, size of the window: $5, k=0.3, \mu=0.4$. Iterations $0,50,100,150,180,210$.

the following term $\left[\int_{\Omega}\langle x-y, \nabla \Phi(y)\rangle e^{-\|x-y\|_{2}^{2} / d^{2}} H_{\epsilon}(\Phi(y)+\right.$ l) $H_{\epsilon}(l-\Phi(y)) d y \bar{n}$ can be interpreted as a repelling force, which ensures topology preservation.

Remark: We continue here the comparison discussion between our approach and the one proposed by Rochery et al. [25]-[27]. The main difference lies in the formulation and in the implementation. In our approach, the energy is straightforwardly formulated in the level-set framework and the gradient descent PDE in the unknown $\Phi$ is directly obtained from the Euler-Lagrange equation. In Rochery et al.'s approach, the energy is first formulated in terms of curves, then the gradient descent for the curve evolution is obtained with a speed $F$. Only after this step is the model cast in the level-set setting to implement the evolution. The speed $F$ must be accurately computed and extended to all level lines. This is done in a two-step algorithm: the speed $F$ is first evaluated in several steps by explicitly extracting the curve points from the zero-level line of $\Phi$, (interpolation is also needed). Finally, the speed $F$ on the contour is extended to all level lines by solving an additional PDE, reinitialization to the distance function being needed as in our approach. Note that in all variational relevant approaches in [1], [25]-[27], [36], and [37], the penalty term is a line integral (more difficult to handle in practice), unlike our case where we have a region integral. To conclude this remark, in Rochery's work, the application is different, devoted to the detection of long and thin objects, such as roads in satellite images and also the function $\Psi\left(\left\|C(p)-C\left(p^{\prime}\right)\right\|\right)$ is different in our model.

\section{EXPERIMENTAL RESULTS}

We conclude the paper by presenting several numerical results and comparisons on both synthetic and real images in 2-D and 3-D. Our experimental tests and images are similar to those

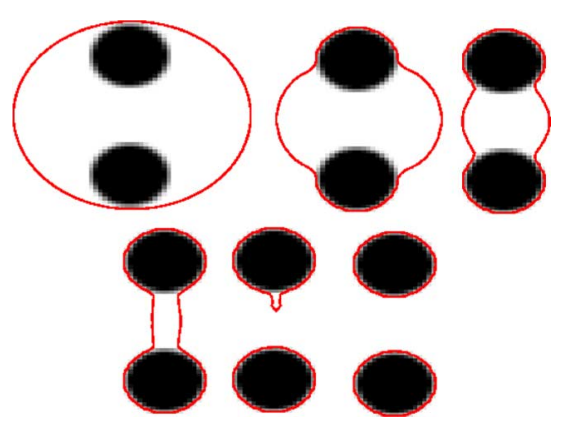

Fig. 6. Segmentation of the synthetic image with two disks when no topological constraint is enforced with the same parameters as above: the contour has split into two components. Iterations 0, 50, 100, 140, 190, 210.

from the related prior work by Han et al. [15], [16] and by Sundaramoorthi and Yezzi [36], [37] and can, thus, be considered as comparisons as well. In addition, we also show comparisons with images used in these prior works, and we can conclude that our proposed method qualitatively performs in a similar way, but by a different approach. The experiments have been performed on a $2.21-\mathrm{GHz}$ Athlon with $1.00 \mathrm{~GB}$ of RAM. Parameters are given together with a verification of the topology of the final contours (surfaces), as suggested by one of the unknown referees. We also show comparisons with the geodesic active contour model (without topology constraint), and we will see that the use of the penalty term improves the accuracy of the segmentation. Many of our numerical results are applied to medical images; however, we do not really use any anatomical or physiological information. By the illustrated results, we only wish to demonstrate how the method performs in practice; for more accurate segmentations in specialized medical applications, the methods and the parameters must be modified and adapted to such applications. For any of the tests, the parameter $\mu$ can be simply set to zero in order not to constrain the topology.

We start with a synthetic image representing two discs in Fig. 5 (similar to tests performed in related prior works by Han et al. [15], [16] and by Sundaramoorthi and Yezzi [36], [37]). We aim at segmenting these two discs while maintaining the same topology throughout the process, which means that we expect to get one path-connected component. The initialization is made with a signed-distance function $\Phi^{0}$ that encloses the two balls and which is the cone defined by: $\Phi^{0}(x, y)=\sqrt{(x-48 h)^{2}+(y-50 h)^{2}}-26$. A comparison is made (Fig. 6) with the results obtained in the case where no topological constraint is enforced.

The second example in Fig. 7 was taken from [16]. We can thus compare our method with the result obtained in [16] on the same image (and shown in the last image from Fig. 7, courtesy of Han and Prince). One can observe that for our test, the two middle fingers touch so with the use of classical geodesic active contours, the evolving contour is going to merge and a hole will then appear (see Fig. 8), which is undesirable. With our method, the repelling forces prevent the curve from merging, as in [16] and [15].

The method has been tested on complex slices of the brain. We can see that the method enables us to get the details of the brain envelope as in Fig. 9 without merges. In Fig. 10, this is 

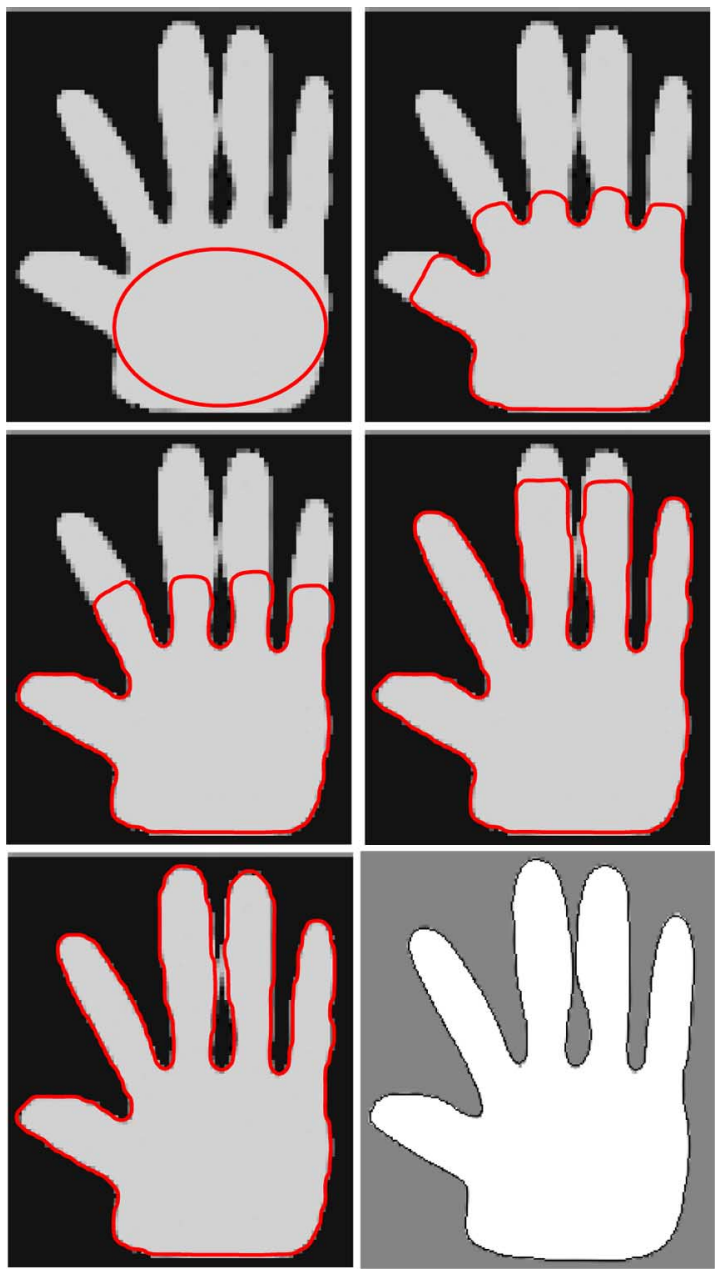

Fig. 7. Segmentation of the hand image taken from [16] by the proposed method with the topology constraint. The result is obtained with the following parameters: $h=1, \tau=0.5, l=1$, size of the window: $7, k=-0.2$, $\mu=0.2$. Iterations $0,150,250,500,600$ and result obtained in [14]-[16] by Han et al.
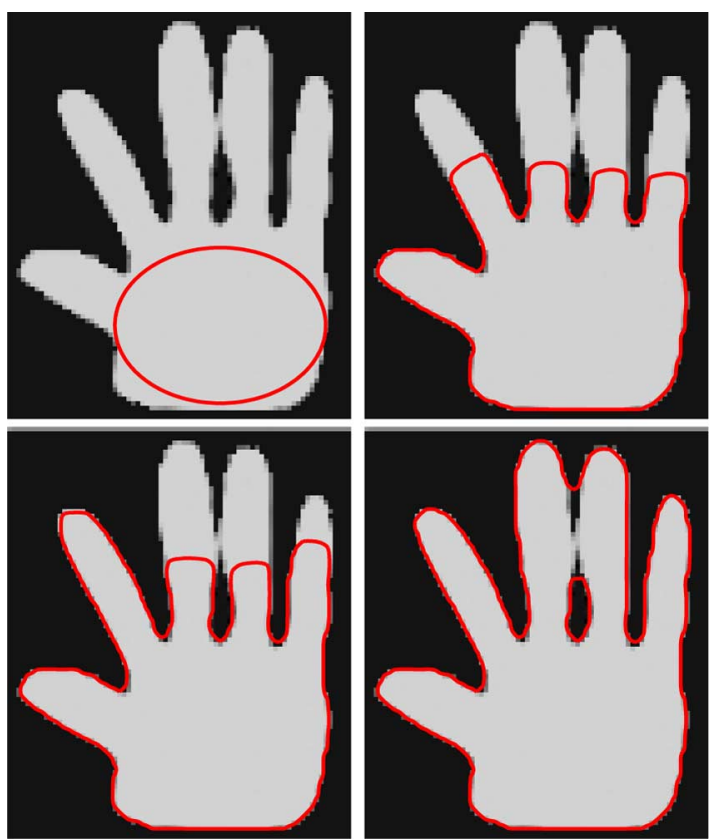

Fig. 8. Steps of the process when no topological constraint is enforced, with the same parameters.

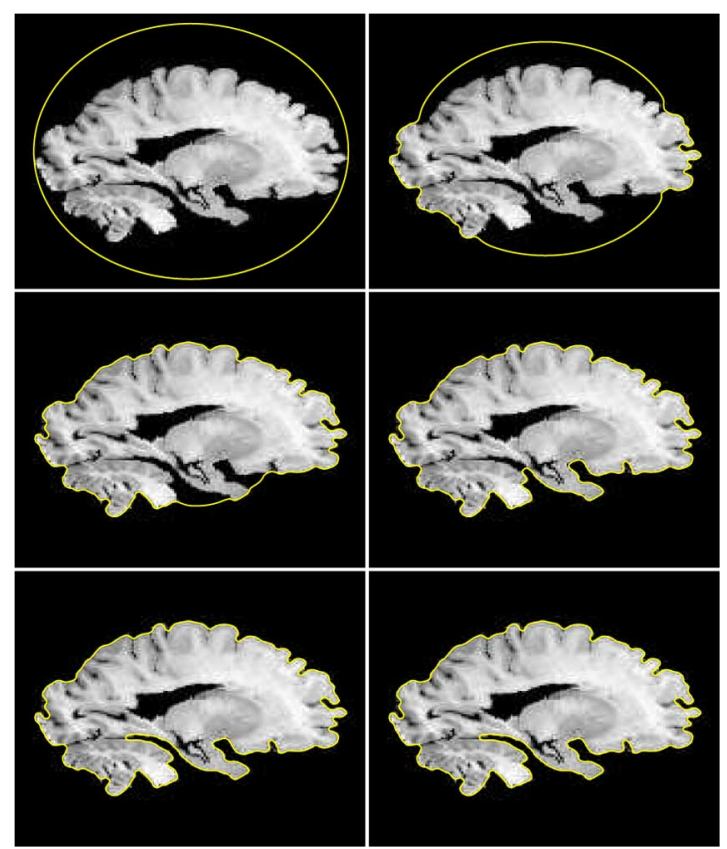

Fig. 9. Obtained evolution when topological constraints are enforced. Iterations $0,100,250,450,720,800$
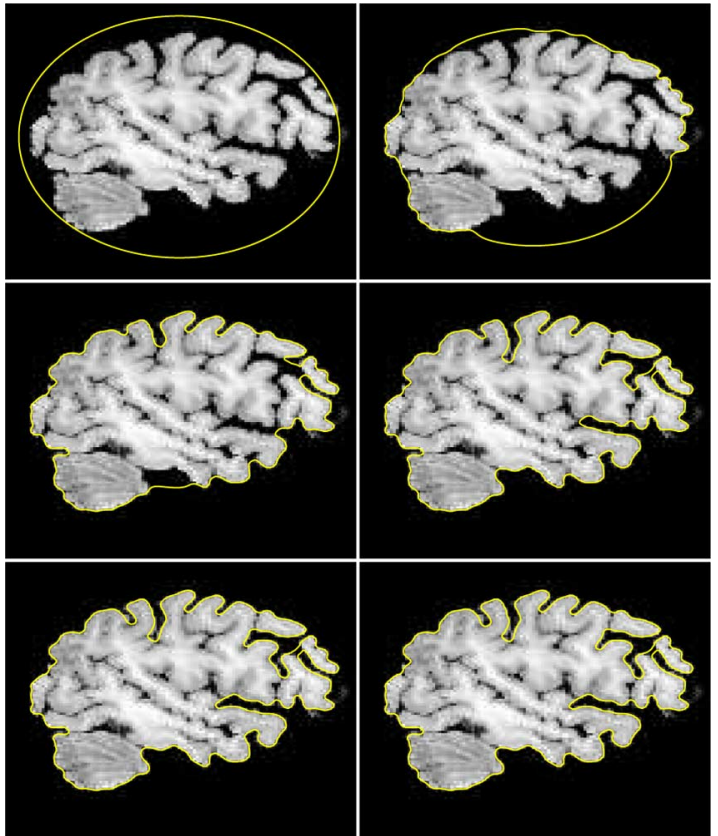

Fig. 10. Results obtained with topological constraints. Iterations 0, 50, 200, $400,480,700$

even more complicated since the slice shows two disconnected parts that are very close to each other.

Fig. 11 illustrates the case where the initial condition is made of two disjoint closed curves. We expect to have both curves evolving without merging. This test is similar to tests presented in related prior work by Han et al. [15], [16] and Sundaramoorthi and Yezzi [36], [37].

We also show in Figs. 12-15 additional segmentation results together with comparisons with the geodesic active contour model. These visually show that the use of the topological penalty term improves the accuracy of the segmentation results. 


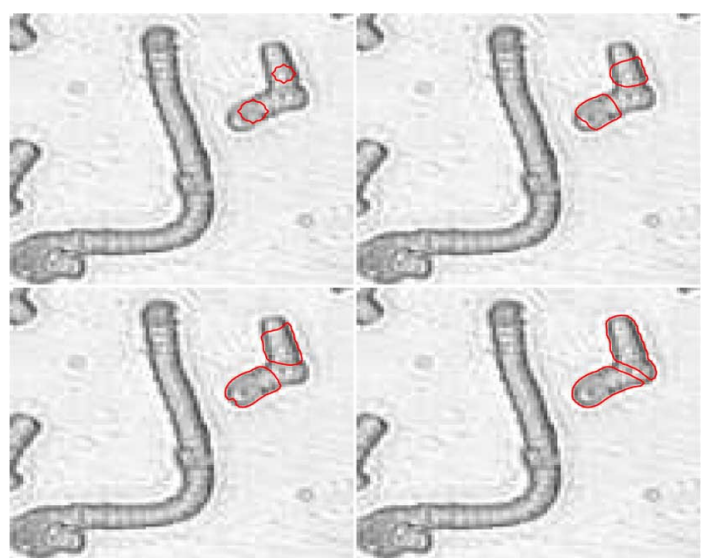

Fig. 11. Segmentation of two blood cells that are very close to each other. Iterations $0,50,100$, and 360 .

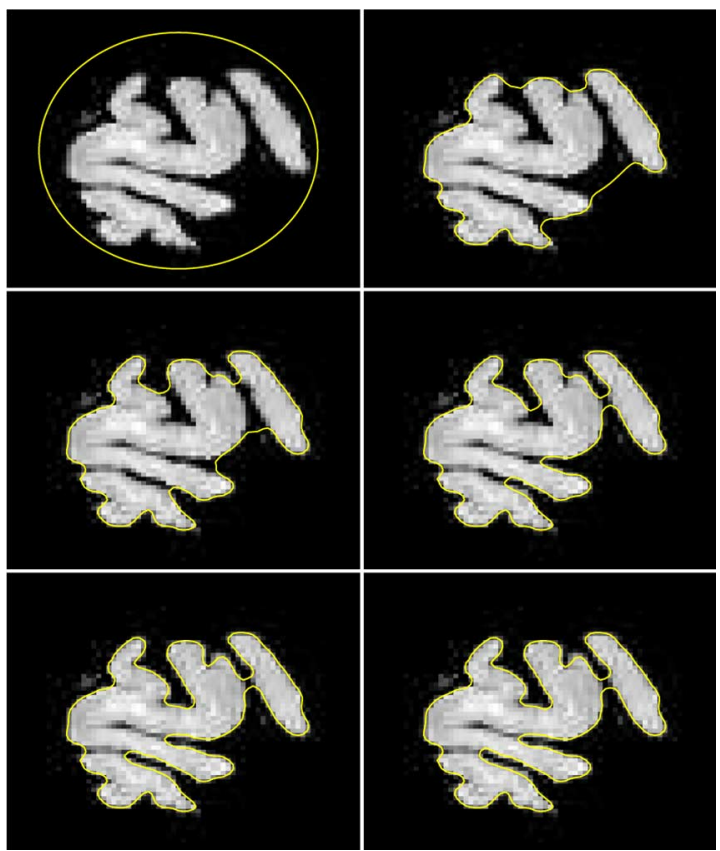

Fig. 12. Segmentation with topology constraint. Iterations $0,100,150,200$, 250 , and 300

A last and more extreme 2-D synthetic example (again included only for the purpose of illustration) is given in Fig. 16. The considered synthetic image is a black crown in a white background. The process is initialized with a curve inside the crown. The topological constraints are applied so that the contour does not split into two contours.

We conclude the paper by presenting 3-D experiments. We first consider two black spheres in a white background as depicted in Fig. 17, top. The process is initialized with a larger sphere that encloses the two small spheres. We apply our topology-preserving method and get the final result shown in Fig. 17, bottom.

The second 3-D image consists of some slices of the brain. We start with an initial condition such that the zero level set encloses the brain. We apply both the topology-preserving model and the classical geodesic active contour model. Owing to data noise/ quality impairment of the image, there exists handles in the gray

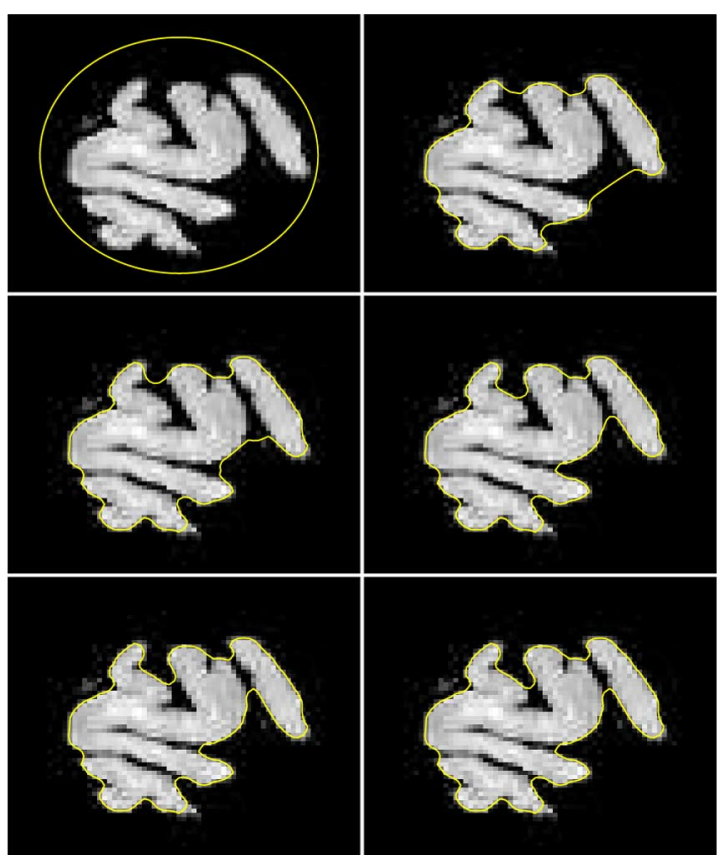

Fig. 13. Segmentation without topology constraint. Iterations $0,100,150,200$, 250,300 .

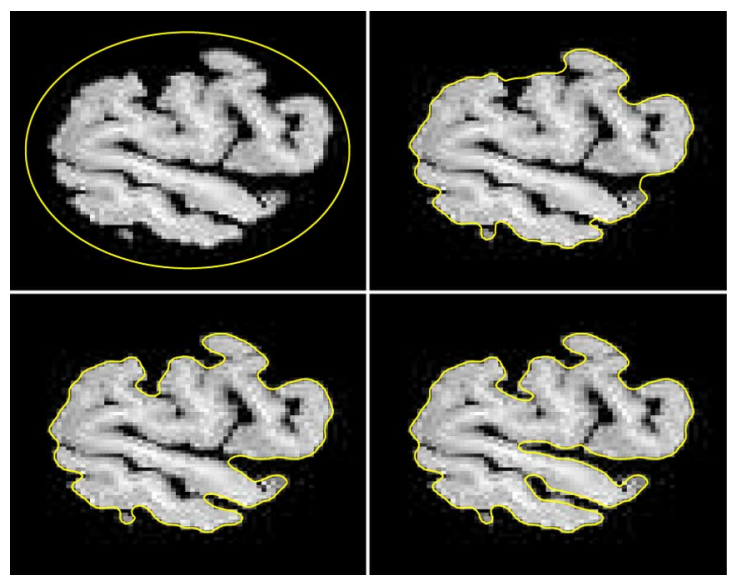

Fig. 14. Segmentation with topology constraint. Iterations 0, 100, 200, and 400
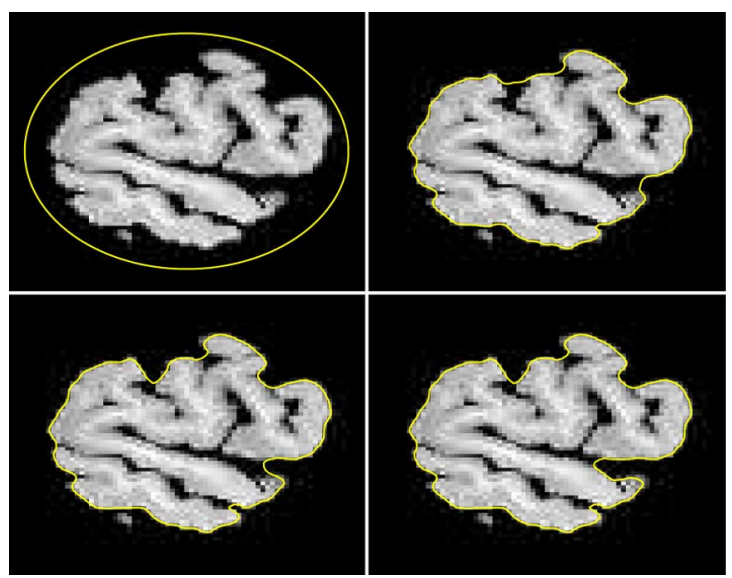

Fig. 15. Segmentation without topology constraint. Iterations $0,100,200$, and 400 .

matter segmentation, which is not consistent with the known spherical topology. When no topological constraint is enforced, 


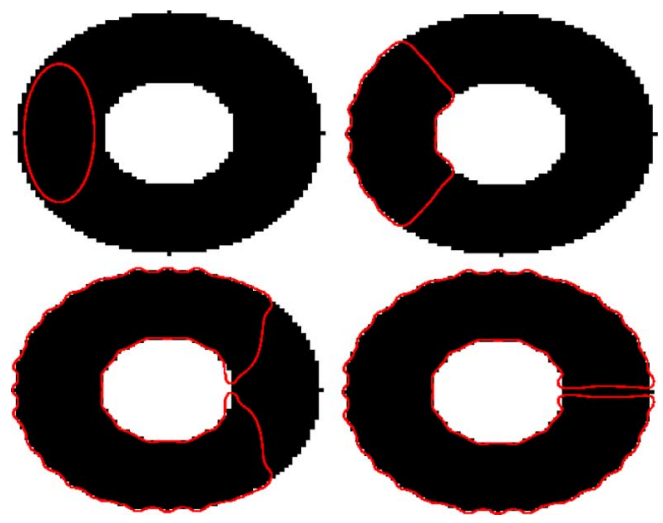

Fig. 16. Segmentation of a torus with topology constraint (equivalent to the disk) with the following parameters: $h=1, \tau=0.5, l=1$, size of the window: $5, k=-0.3, \mu=0.4$. Iterations $0,50,300$, and 500 .

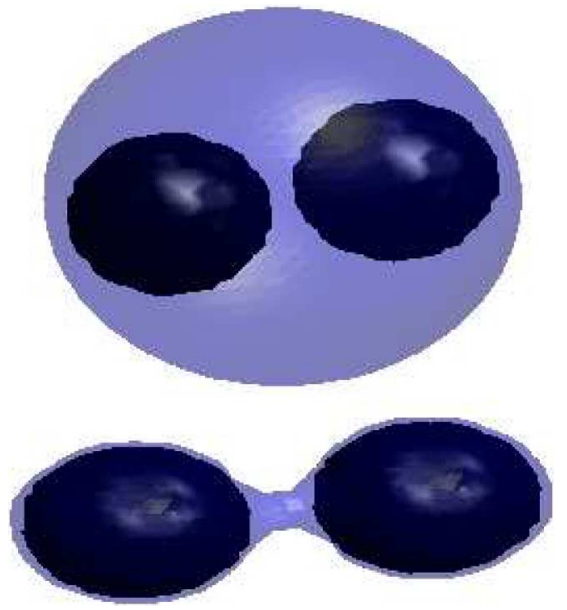

Fig. 17. Initialization by the function $\Phi^{0}(x, y, z)=$ $\sqrt{(x-25 h)^{2}+(y-25 h)^{2}+(z-25 h)^{2}}-18$. Top, in light blue, the zero level set. Bottom, in light blue, the final result.

the handle appears on the top right corner in Fig. 19, which is not the case when the constraint is integrated in the model, Fig. 18.

We end this section with a 3-D image representing an entire brain, in Figs. 20 and 21. We expect to get a shape topologically equivalent to the sphere. The details of the lobes are clearly extracted without contact points, there is no handle or anatomically incorrect structures. The spherical topology is kept throughout the process.

\section{A. Numerical Verification and Validation of the Topology Constraint}

For our 2-D experiments, by visual inspection only we can see that the topology constraint has been correctly imposed. However, for the 3-D results, visual inspection alone is not sufficient to verify that the correct desired topology has been achieved.

To make sure of the algorithm efficiency, we have computed, for each 3-D example, the Euler characteristic $\chi$ of the obtained closed surface (extracted using the 'isosurface' function of Matlab). The Euler characteristic is a topological invariant which, with the knowledge of the orientation of the considered closed surface, allows to (uniquely) topologically classify this surface.
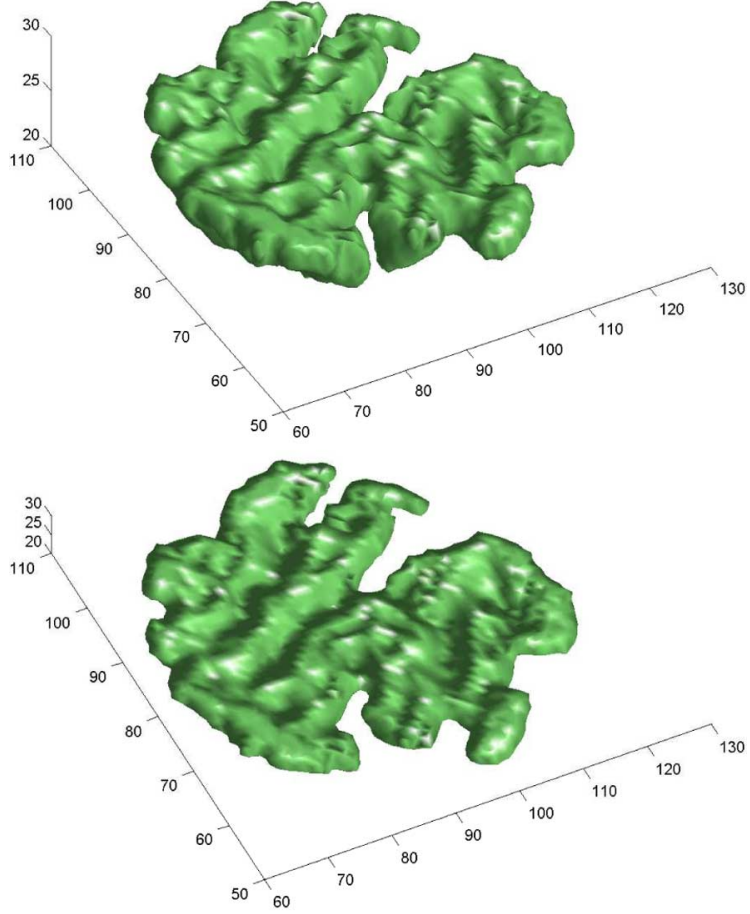

Fig. 18. Two views of the final result when the topology constraint is enforced.
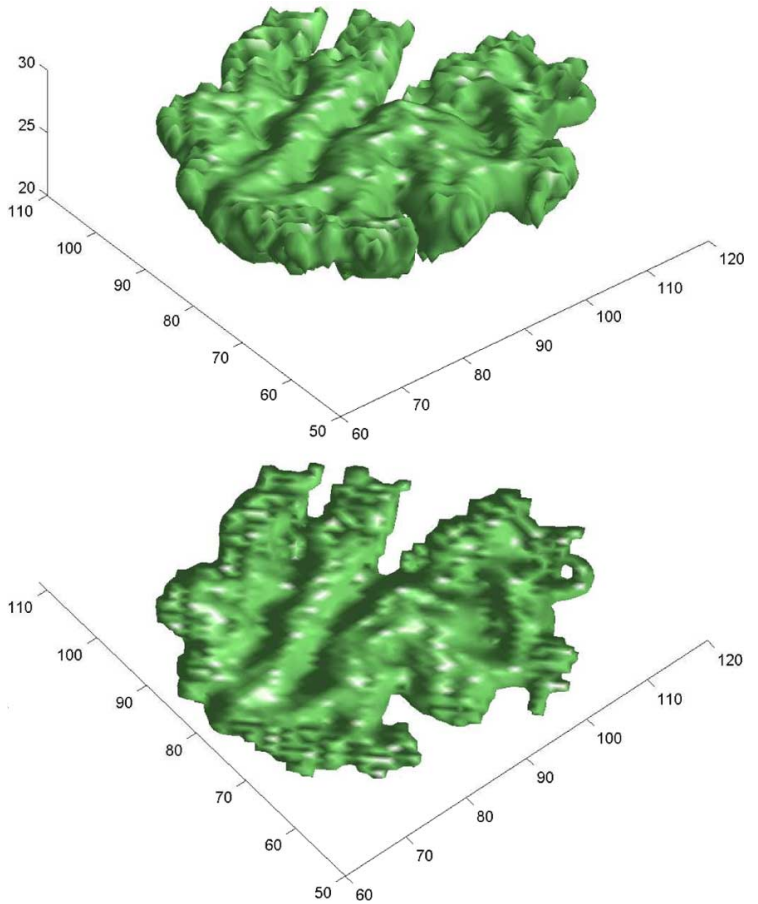

Fig. 19. Two views of the final result when no topology constraint is applied.

In the case of polyhedra, it is classically defined as follows:

$$
\chi=V-E+F
$$

with $V$ the number of vertices, $E$ the number of edges and $F$ the number of faces.

It is known that for any polyhedron homeomorphic to a sphere, the Euler characteristic is equal to 2 . Thus, as we aim at getting surfaces topologically equivalent to a sphere, 


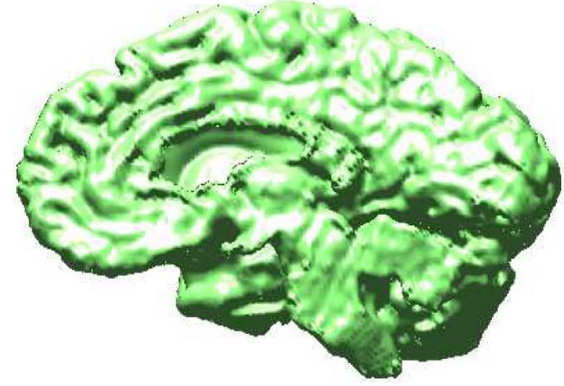

Fig. 20. Top view of the final result.

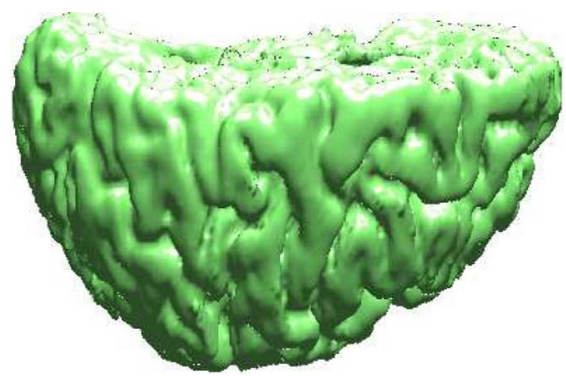

Fig. 21. Front view of the final result.

we can expect to get a Euler characteristic equal to 2 in our experiments.

In the example of the two spheres, we have obtained

$$
\left\{\begin{array}{l}
V=2732 \\
E=8190, \text { which gives } \chi=2 \\
F=5460 .
\end{array}\right.
$$

In the example related to Fig. 18, we have obtained

$$
\left\{\begin{array}{l}
V=6828 \\
E=20478, \text { which gives again } \chi=2 \\
F=13652 .
\end{array}\right.
$$

In comparison, the computation of the Euler characteristic in the example related to Fig. 19 gives

$$
\left\{\begin{array}{l}
V=6062 \\
E=18186, \text { that is } \chi=0 \\
F=12124 .
\end{array}\right.
$$

This result is consistent with the theory. The Klein bottle and the torus are the only closed surfaces with a Euler characteristic equal to 0 . Due to the hole in the top right part of the gray matter, the obtained surface is topologically equivalent to a torus.

We conclude with the image of the whole brain corresponding to Figs. 20 and 21. We have obtained

$$
\left\{\begin{array}{l}
V=63626 \\
E=190872, \text { that is } \chi=2 \\
F=127248 .
\end{array}\right.
$$

\section{B. Discussion on the Choice and the Dependence of the Parameters}

The window size in the Gaussian function is small due to computational cost and is related to the parameter $l$ (kept equal to 1 in all our calculations). We choose the window size to be $5 \times 5$ or $7 \times 7$, while the parameter $d$ is always defined as $d=l+3$. The values for $\mu$ are chosen small, inside the interval $[0.2,0.4]$, so that we do not have the penalty as the dominant term. Still, with such small $\mu$ values, the topological constraint is satisfied. The value of $\triangle t=\tau$ is chosen function of the $C F L$ condition of the numerical scheme, and, finally, the parameter $k$ has the same meaning as in other deformable balloon models (to speed up the evolution, and to force the motion inward or outward). The distance between two close curves is thus dependent on the values of $l, d$ and of the Gaussian window size. With mesh refinement near the contour, it would be possible to use smaller such values, so that the curves can touch each other but without changing the topology. This may be desirable for more accurate segmentations of the cortical surface.

\section{CONCLUSION}

In this paper, we have addressed the issue of topology preservation in segmentation problems. We have proposed a geodesicactive-contour-based model that globally integrates a topological constraint. This topological constraint is based on a geometrical observation and can be seen as a repelling force in the evolution problem. In terms of computational cost, the use of an additive operator splitting (AOS) scheme leads to solve two (three) decoupled tridiagonal linear subsystems. The computation time could be reduced by parallelizing the code even if in most 2-D cases, computation time is of the order of a minute. The topological constraint is easy to compute since we have restricted the integration domain to a small window centered on the current point. Regarding the parameters, the CFL conditions enable us to have an estimate of parameters $\tau, k$, and $\mu$. The level parameter $l$ has been chosen equal to 1 in all the tests in order not to constrain too much the model and to preserve the fine details of the boundaries. It may be interesting and useful to apply the proposed penalty term to other segmentation approaches, including region-based methods.

\section{APPENDIX A}

\section{DERIVATION OF THE EULER-LAGRANGE EQUATIONS}

In the sequel, the Euler-Lagrange equation associated to problem (5) is computed. It can be formally obtained as follows. Let us set $\Upsilon(\nu)=f(\nu)+\mu e(\nu)$, with $f(\nu)=F(\Phi+\nu \Psi)$ and $e(\nu)=E(\Phi+\nu \Psi), \nu$ being a small parameter and $\Psi$ a function like $\Phi$. The minimizer $\Phi$ will satisfy $\Upsilon^{\prime}(0)=0=f^{\prime}(0)+\mu e^{\prime}(0)$ for all functions $\Psi$. One has

$$
\begin{aligned}
e(\nu)=\int_{\Omega} \int_{\Omega}\left[\begin{array}{l}
G\left(\|x-y\|_{2}^{2}\right) \\
\cdot
\end{array}\right. & g(\nabla \Phi(x)+\nu \nabla \Psi(x), \nabla \Phi(y)+\nu \nabla \Psi(y)) \\
& \cdot h(\Phi(x)+\nu \Psi(x)) h(\Phi(y)+\nu \Psi(y))] d x d y
\end{aligned}
$$

and a simple calculus gives

$$
\begin{aligned}
e^{\prime}(\nu)=\int_{\Omega} \int_{\Omega}[ & G\left(\|x-y\|_{2}^{2}\right) \\
& \cdot g(\nabla \Phi(x)+\nu \nabla \Psi(x), \nabla \Phi(y)+\nu \nabla \Psi(y)) \\
& \cdot\left(h^{\prime}(\Phi(x)+\nu \Psi(x)) h(\Phi(y)+\nu \Psi(y)) \Psi(x)\right. \\
& +h(\Phi(x)+\nu \Psi(x)) h^{\prime}(\Phi(y) \\
& +\nu \Psi(y)) \Psi(y))] d x d y
\end{aligned}
$$




$$
\begin{aligned}
+\int_{\Omega} \int_{\Omega}[ & G\left(\|x-y\|_{2}^{2}\right) h(\Phi(x)+\nu \Psi(x)) \\
& \cdot h(\Phi(y)+\nu \Psi(y))\left(\left\langle\nabla_{\left(z_{1}, z_{2}\right)} g(\nabla \Phi(x)\right.\right. \\
& +\nu \nabla \Psi(x), \nabla \Phi(y)+\nu \nabla \Psi(y)), \nabla \Psi(x)\rangle \\
& +\left\langle\nabla_{\left(z_{3}, z_{4}\right)} g(\nabla \Phi(x)+\nu \nabla \Psi(x), \nabla \Phi(y)\right. \\
& +\nu \nabla \Psi(y)), \nabla \Psi(y)\rangle)] d x d y .
\end{aligned}
$$

Then see the equation shown at the bottom of the page.

We can switch $x$ and $y$ in the first integral and we get as first component

$$
\begin{gathered}
\int_{\Omega} \int_{\Omega}\left[G\left(\|x-y\|_{2}^{2}\right) g(\nabla \Phi(x), \nabla \Phi(y))\right. \\
\left.\cdot h^{\prime}(\Phi(x)) h(\Phi(y)) \Psi(x)\right] d x d y \\
+\int_{\Omega} \int_{\Omega}\left[G\left(\|x-y\|_{2}^{2}\right) g(\nabla \Phi(y), \nabla \Phi(x))\right. \\
\left.\cdot h^{\prime}(\Phi(x)) h(\Phi(y)) \Psi(x)\right] d y d x .
\end{gathered}
$$

Assuming that we can interchange the order of integration, we get as first component

$$
\begin{gathered}
\int_{\Omega}\left\{\int _ { \Omega } \left[G\left(\|x-y\|_{2}^{2}\right) g(\nabla \Phi(x), \nabla \Phi(y))\right.\right. \\
\left.\left.\cdot h^{\prime}(\Phi(x)) h(\Phi(y))\right] d y\right\} \Psi(x) d x \\
+\int_{\Omega}\left\{\int _ { \Omega } \left[G\left(\|x-y\|_{2}^{2}\right) g(\nabla \Phi(y), \nabla \Phi(x))\right.\right. \\
\left.\left.\cdot h^{\prime}(\Phi(x)) h(\Phi(y))\right] d y\right\} \Psi(x) d x .
\end{gathered}
$$

For the second component, the same reasoning is applied. We switch $x$ and $y$ in the second part of the component. Assuming once again that we can interchange the order of integration, we have

$$
\begin{aligned}
& \int_{\Omega}\left\{\int_{\Omega}[G\right.\left(\|x-y\|_{2}^{2}\right) h(\Phi(x)) h(\Phi(y)) \\
&\left.\left.\cdot\left\langle\nabla_{\left(z_{1}, z_{2}\right)} g(\nabla \Phi(x), \nabla \Phi(y)), \nabla \Psi(x)\right\rangle\right] d x\right\} d y \\
&+\int_{\Omega}\left\{\int _ { \Omega } \left[G\left(\|x-y\|_{2}^{2}\right) h(\Phi(x)) h(\Phi(y))\right.\right. \\
&\left.\left.\cdot\left\langle\nabla_{\left(z_{3}, z_{4}\right)} g(\nabla \Phi(y), \nabla \Phi(x)), \nabla \Psi(x)\right\rangle\right] d x\right\} d y .
\end{aligned}
$$

Integrating by parts with respect to $x$, interchanging the order of integration and setting the necessary boundary conditions to zero, we obtain

$$
\begin{aligned}
& e^{\prime}(0)= \int_{\Omega}\left\{\int_{\Omega} G\left(\|x-y\|_{2}^{2}\right) g(\nabla \Phi(x), \nabla \Phi(y))\right. \\
&\left.\cdot h^{\prime}(\Phi(x)) h(\Phi(y)) d y\right\} \Psi(x) d x \\
&+\int_{\Omega}\left\{\int_{\Omega} G\left(\|x-y\|_{2}^{2}\right) g(\nabla \Phi(y), \nabla \Phi(x))\right. \\
&-\int_{\Omega}\left\{\int_{\Omega} \operatorname{div}\left(G\left(\|x-y\|_{2}^{2}\right) h(\Phi(x)) h(\Phi(y)) h(\Phi(y)) d y\right\} \Psi(x) d x\right. \\
& \cdot\left.\left.\cdot \nabla_{\left(z_{1}, z_{2}\right)} g(\nabla \Phi(x), \nabla \Phi(y))\right) d y\right\} \Psi(x) d x \\
&- \int_{\Omega}\left\{\int _ { \Omega } \operatorname { d i v } \left(G\left(\|x-y\|_{2}^{2}\right) h(\Phi(x)) h(\Phi(y))\right.\right. \\
&\left.\left.\cdot \nabla_{\left(z_{3}, z_{4}\right)} g(\nabla \Phi(y), \nabla \Phi(x))\right) d y\right\} \Psi(x) d x
\end{aligned}
$$

for all $\Psi$.

$$
\begin{aligned}
e^{\prime}(0)= & \int_{\Omega} \int_{\Omega}\left[G\left(\|x-y\|_{2}^{2}\right) g(\nabla \Phi(x), \nabla \Phi(y))\left(h^{\prime}(\Phi(x)) h(\Phi(y)) \Psi(x)+h(\Phi(x)) h^{\prime}(\Phi(y)) \Psi(y)\right)\right] d x d y \\
+ & \int_{\Omega} \int_{\Omega}\left[G\left(\|x-y\|_{2}^{2}\right) h(\Phi(x)) h(\Phi(y))\right. \\
& \left.\cdot\left(\left\langle\nabla_{\left(z_{1}, z_{2}\right)} g(\nabla \Phi(x), \nabla \Phi(y)), \nabla \Psi(x)\right\rangle+\left\langle\nabla_{\left(z_{3}, z_{4}\right)} g(\nabla \Phi(x), \nabla \Phi(y)), \nabla \Psi(y)\right\rangle\right)\right] d x d y
\end{aligned}
$$


Using (3), we deduce that the obtained Euler-Lagrange equation formally is

$$
\begin{aligned}
&-\delta_{\epsilon}(\Phi) \operatorname{div}\left(g(|\nabla I|) \frac{\nabla \Phi}{|\nabla \Phi|}\right) \\
&+\mu\left\{\int _ { \Omega } \left[G\left(\|x-y\|_{2}^{2}\right)(g(\nabla \Phi(x), \nabla \Phi(y))\right.\right. \\
&+\left.g(\nabla \Phi(y), \nabla \Phi(x))) h^{\prime}(\Phi(x)) h(\Phi(y))\right] d y \\
&-\int_{\Omega}\left[\operatorname { d i v } \left(G\left(\|x-y\|_{2}^{2}\right) h(\Phi(x)) h(\Phi(y))\right.\right. \\
&\left.\left.\cdot \nabla_{\left(z_{1}, z_{2}\right)} g(\nabla \Phi(x), \nabla \Phi(y))\right)\right] d y \\
&-\int_{\Omega}\left[\operatorname { d i v } \left(G\left(\|x-y\|_{2}^{2}\right) h(\Phi(x)) h(\Phi(y))\right.\right. \\
&\left.\left.\left.\cdot \nabla_{\left(z_{3}, z_{4}\right)} g(\nabla \Phi(y), \nabla \Phi(x))\right)\right] d y\right\}=0
\end{aligned}
$$

with associated boundary conditions.

After some intermediate computations, we eventually obtain

$$
\begin{gathered}
2 H_{\epsilon}(\Phi(x)+l) H_{\epsilon}(l-\Phi(x)) \\
\cdot \int_{\Omega}\left[-2 \frac{\mu}{d^{2}}\left(\left(x_{1}-y_{1}\right) \frac{\partial \Phi}{\partial y_{1}}(y)+\left(x_{2}-y_{2}\right) \frac{\partial \Phi}{\partial y_{2}}(y)\right)\right. \\
\left.\cdot e^{-\|x-y\|_{2}^{2} / d^{2}} H_{\epsilon}(\Phi(y)+l) H_{\epsilon}(l-\Phi(y))\right] d y \\
-\delta_{\epsilon}(\Phi) \operatorname{div}\left(g(|\nabla I|) \frac{\nabla \Phi}{|\nabla \Phi|}\right)=0 .
\end{gathered}
$$

\section{APPENDIX B}

\section{DESCRIPTION OF THE NUMERICAL ALGORITHM}

For the discretization stage, we use an AOS scheme, and we follow Weickert et al. (see [41] for more details), which allows the decomposition of the initial 2-D problem into two 1-D subproblems. This splitting-based scheme is easy to implement and requires a linear computational cost at each step.

To simplify the notation, we use a vectorial representation of the function $\Phi$ via a concatenation of the rows (respectively columns) of the associated matrix. Thus, $\Phi \in \mathbb{R}^{N \times M}$ where $N$ is the number of lines and $M$ the number of columns. The center of gravity of a pixel $i$ coincides with the node of the mesh of coordinates $x_{i}$. Let us denote by $h$ the space step (assumed to be equal to 1) and $\tau$ the time step. Let $\Phi_{i}^{n}$ be an approximation of $\Phi\left(x_{i}, t_{n}\right)=\Phi\left(x_{i}, n \tau\right)$. The considered discretization of the problem is as follows:

$$
\begin{aligned}
\Phi^{n+1}= & \frac{1}{2} \sum_{l \in\{x, y\}}\left(I d-2 \tau A_{l}\left(\Phi^{n}\right)\right)^{-1} \\
& \cdot\left[\Phi^{n}+4 \frac{\mu \tau}{d^{2}} H_{\epsilon}\left(\Phi^{n}+l\right) H_{\epsilon}\left(l-\Phi^{n}\right)\right.
\end{aligned}
$$

$$
\begin{aligned}
& \cdot \int_{\Omega}\left(\left\langle x-y, \nabla \Phi^{n}(y)\right\rangle e^{-\|x-y\|_{2}^{2} / d^{2}} H_{\epsilon}\left(\Phi^{n}(y)+l\right)\right. \\
& \left.\left.\cdot H_{\epsilon}\left(l-\Phi^{n}(y)\right)\right) d y+\tau k g(|\nabla I|)\left|\nabla \Phi^{n}\right|\right]
\end{aligned}
$$

with the entries of $A_{l}\left(\Phi^{n}\right)$ defined by

$$
a_{i j_{l}}= \begin{cases}\left|\nabla \Phi_{i}^{n}\right| \frac{2}{\left(\frac{|\nabla \Phi|}{g(|\nabla I|)}\right)_{i}^{n}+\left(\frac{|\nabla \Phi|}{g(|\nabla I|)}\right)_{j}^{n}} & \text { if } j \in \wedge_{l}(i) \\ -\left|\nabla \Phi_{i}^{n}\right| \sum_{m \in \wedge_{l}(i)} \frac{2}{\left(\frac{|\nabla \Phi|}{g(|\nabla I|)}\right)_{i}^{n}+\left(\frac{|\nabla \Phi|}{g(|\nabla I|)}\right)_{m}^{n}} & \text { if } i=j, \\ 0, & \text { otherwise }\end{cases}
$$

and where $\wedge_{l}(i)$ represents the neighboring pixels of $i$ with respect to direction $l$.

The AOS scheme can be rewritten by means of the matrices $B_{l}\left(\Phi^{n}\right)=I d-2 \tau A_{l}\left(\Phi^{n}\right), l \in\{x, y\}$. We obtain two disconnected linear systems that are both tridiagonal if one reorders the nodes of the mesh in a different way for the second system. The matrices $B_{l}$ are tridiagonal, strictly diagonally dominant (which ensures uniqueness of the solution of both linear systems thanks to Gershgorin's theorem for instance) and lead to systems that can be solved computing Thomas algorithm with linear complexity.

Weickert et al. [41] prove that all the coefficients of matrices $B_{l}^{-1}\left(\Phi^{n}\right)$ are non-negative and that the sum of the elements of each row of $B_{l}^{-1}\left(\Phi^{n}\right)$ is equal to one. Thus, if we neglect the topological constraints and the component $k g(|\nabla I|)|\nabla \Phi|$, it implies that each element of $\Phi^{n+1}$ is computed from a convex combination of components of $\Phi^{n}$. The discrete minimum-maximum principle applies and stability is, therefore, guaranteed for any time step.

Regarding the constant motion $k g(|\nabla I|)|\nabla \Phi|$, the function $g(|\nabla I|)$ controls this term and annihilates the action of the force when the curve is approaching edge points. A classical discretization of the gradient can lead to the creation of loops. The entropy condition [34] prevents the curve from propagating where it has already been and thus avoids loop formation. The resulting discretization of the gradient comes from the hyperbolic conservation laws and can be found in [34]

$$
\begin{gathered}
|\nabla \Phi|_{i}^{n 2} \simeq\left[\max \left(D^{-x} \Phi_{i}^{n}, 0\right)^{2}+\min \left(D^{+x} \Phi_{i}^{n}, 0\right)^{2}\right. \\
\left.+\max \left(D^{-y} \Phi_{i}^{n}, 0\right)^{2}+\min \left(D^{+y} \Phi_{i}^{n}, 0\right)^{2}\right], \text { if } k<0 \\
{\left[\max \left(D^{+x} \Phi_{i}^{n}, 0\right)^{2}+\min \left(D^{-x} \Phi_{i}^{n}, 0\right)^{2}\right.} \\
\left.+\max \left(D^{+y} \Phi_{i}^{n}, 0\right)^{2}+\min \left(D^{-y} \Phi_{i}^{n}, 0\right)^{2}\right], \text { if } k>0
\end{gathered}
$$

using the classical finite-difference operators $D^{+x} \Phi_{i}=\Phi_{i+1}$ $\Phi_{i}$ and $D^{-x} \Phi_{i}=\Phi_{i}-\Phi_{i-1}$ in 1-D.

As for the topological constraint, we have used a $\mathcal{C}^{\infty}$ regularization of the Heaviside function (see [7]) defined by

$$
H_{\epsilon}(z)=\frac{1}{2}\left(1+\frac{2}{\pi} \arctan \left(\frac{z}{\epsilon}\right)\right)
$$


The partial derivatives are approximated using central finite difference schemes. While the topological constraint introduced in the modeling enables us to use the level-set framework to track interfaces whose topology must be preserved, the main drawback is computational cost due to the nonlocal term appearing in the evolution equation. This limitation can be overcome by slightly simplifying the issue: instead of computing the nonlocal term over the whole grid, we compute it over a square centered on the current point. The window size depends on the level parameter $l$. This simplification looks realistic since we are mainly interested in the behavior of $\Phi$ in a neighborhood of the current point.

Last, we have assumed that $\Phi$ is a signed-distance function. Thus, we need to periodically apply a reinitialization process. We have used the scheme obtained by Russo and Smereka in [28], defined by the first equation at the bottom of the page with $D_{i j}$, the distance of node $(i, j)$ from the interface and where (see the second equation shown at the bottom of the page).

In the 3-D case, the numerical scheme is defined in a same manner as in the 2-D case, and thus avoids loop formation (see the third equation shown at the bottom of the page) with $A_{l}\left(\Phi^{n}\right)$ defined as previously done and $\langle\cdot, \cdot\rangle$ the Euclidean scalar product in $\mathbb{R}^{3}$.

\section{ACKNOWLEDGMENT}

The authors would like to thank J.-M. Morel for his very useful suggestions on this project, C. Gout for carefully reading the manuscript, and J. Prince for suggesting references [36] and [37]. They would also like to thank the anonymous referees for their very useful remarks and constructive suggestions that greatly helped to improve the presentation of the manuscript.

\section{REFERENCES}

[1] O. Alexandrov and F. Santosa, "A topology-preserving level set method for shape optimization," J. Comput. Phys., vol. 204, no. 1, pp. 121-130, 2005.

[2] L. Alvarez, P.-L. Lions, and J.-M. Morel, "Image selective smoothing and edge detection by nonlinear diffusion," SIAM J. Numer. Anal, vol. 29, no. 3, pp. 845-866, 1992.

[3] G. Aubert and P. Kornprobst, Mathematical Problems in Image processing: Partial Differential Equations and the Calculus of Variations. New York: Springer Verlag, 2002.

[4] V. Caselles, F. Catté, C. Coll, and F. Dibos, "A geometric model for active contours in image processing," Numer. Math., vol. 66, pp. 1-31, 1993.

[5] V. Caselles, R. Kimmel, and G. Sapiro, "Geodesic active contours," Int. J. Comput. Vis., vol. 22, no. 1, pp. 61-87, 1993.

[6] T. Cecil, "Numerical methods for partial differential equations involving discontinuities," Ph.D. dissertation, Dept. Math., Univ. California, Los Angeles, 2003.

$$
\Phi_{i, j}^{n+1}= \begin{cases}\Phi_{i, j}^{n}-\frac{\Delta t}{\Delta x}\left(\operatorname{sgn}\left(\Phi_{i, j}^{0}\right)\left|\Phi_{i, j}^{n}\right|-D_{i, j}\right), & \text { if } \Phi_{i, j}^{0} \Phi_{i-1, j}^{0}<0 \text { or } \Phi_{i, j}^{0} \Phi_{i+1, j}^{0}<0 \text { or } \Phi_{i, j}^{0} \Phi_{i, j-1}^{0}<0 \text { or } \Phi_{i, j}^{0} \Phi_{i, j+1}^{0}<0 \\ \Phi_{i, j}^{n}-\Delta \operatorname{sgn}\left(\Phi_{i, j}^{0}\right) G(\Phi)_{i, j}, & \text { otherwise }\end{cases}
$$

and

$$
\begin{aligned}
G(\Phi)_{i, j}= & {\left[\max \left(\left|\max \left(D_{x}^{-} \Phi_{i, j}, 0\right)\right|^{2},\left|\min \left(D_{x}^{+} \Phi_{i, j}, 0\right)\right|^{2}\right)\right.} \\
& \left.\quad+\max \left(\left|\max \left(D_{y}^{-} \Phi_{i, j}, 0\right)\right|^{2},\left|\min \left(D_{y}^{+} \Phi_{i, j}, 0\right)\right|^{2}\right)-1\right]^{1 / 2}, \text { if } \Phi_{i, j}^{0}>0
\end{aligned}
$$

$$
\begin{aligned}
G(\Phi)_{i, j}= & {\left[\max \left(\left|\min \left(D_{x}^{-} \Phi_{i, j}, 0\right)\right|^{2},\left|\max \left(D_{x}^{+} \Phi_{i, j}, 0\right)\right|^{2}\right)\right.} \\
& \left.\quad+\max \left(\left|\min \left(D_{y}^{-} \Phi_{i, j}, 0\right)\right|^{2},\left|\max \left(D_{y}^{+} \Phi_{i, j}, 0\right)\right|^{2}\right)-1\right]^{1 / 2}, \text { if } \Phi_{i, j}^{0}<0
\end{aligned}
$$

$$
\begin{aligned}
\Phi^{n+1}= & \frac{1}{3} \sum_{l \in\{x, y, z\}}\left(I d-3 \tau A_{l}\left(\Phi^{n}\right)\right)^{-1} \\
& {\left[\Phi^{n}+4 \frac{\mu \tau}{d^{2}} H_{\epsilon}\left(\Phi^{n}+l\right) H_{\epsilon}\left(l-\Phi^{n}\right)\right.} \\
& \left.\cdot \int_{\Omega}\left(\left\langle x-y, \nabla \Phi^{n}(y)\right\rangle e^{-\|x-y\|_{2}^{2} / d^{2}} H_{\epsilon}\left(\Phi^{n}(y)+l\right) H_{\epsilon}\left(l-\Phi^{n}(y)\right)\right) d y+\tau k g(|\nabla I|)\left|\nabla \Phi^{n}\right|\right]
\end{aligned}
$$


[7] T. Chan and L. Vese, "Active contours without edges," IEEE Trans. Image Process., vol. 10, no. 2, pp. 266-277, Feb. 2001.

[8] I. Cohen, L. D. Cohen, and N. Ayache, "Using deformable surfaces to segment 3D images and infer differential structures," Comput., Vis., Graph. Image Process.: Image Understand., vol. 56, no. 2, pp. 242-263, 1992.

[9] L. D. Cohen, E. Bardinet, and N. Ayache, "Surface reconstruction using active contour models," INRIA, 1992, Res. Rep.

[10] L. D. Cohen and R. Kimmel, "Global minimum for active contour models: A minimal path approach," presented at the CVPR, San Fransisco, CA, 1996

[11] A. Dervieux and F. Thomasset, "A finite element method for the simulation of Rayleigh-Taylor instability," Lecture Notes Math., vol. 771, pp. 145-159, 1979.

[12] A. Dervieux and F. Thomasset, "Multifluid incompressible flows by a finite element method," Lecture Notes Phys., vol. 141, pp. 158-163, 1980.

[13] A. Dufour, V. Shinin, S. Tajkhbaksh, N. Guillezn-Aghion, J.-C. OlivoMarin, and C. Zimmer, "Segmenting and tracking fluorescent cells in dynamic 3-D microscopy with coupled active surfaces," IEEE Trans. Image Process., vol. 14, no. 9, pp. 1396-1410, 2005.

[14] X. Han, C. Xu, U. Braga-Neto, and J. L. Prince, "Topology correction in brain cortex segmentation using a multiscale, graph-based algorithm," IEEE Trans. Med. Imag., vol. 21, no. 2, pp. 109-121, Feb. 2002.

[15] X. Han, C. Xu, U. Braga-Neto, and J. L. Prince, "A topology preserving geometric deformable model and its application in brain cortical surface reconstruction," in Geometric Level Set Methods in Imaging, Vis., Graph., S. Osher and N. Paragios, Eds. New York: Springer Verlag, 2003.

[16] X. Han, C. Xu, and J. L. Prince, "A topology preserving level set method for geometric deformable models," IEEE Trans. Pattern Anal. Mach. Intell., vol. 25, no. 6, pp. 755-768, Jun. 2003.

[17] M. Kass, A. Witkin, and D. Terzopoulos, "Snakes: Active contour models," Int. J. Comput. Vis., vol. 1, no. 4, pp. 133-144, 1987.

[18] R. Malladi and J. A. Sethian, "Image processing via level curvature flow," Proc. NAS, vol. 92, no. 15, pp. 7046-7050, 1995

[19] T. McInerney and D. Terzopoulos, "Deformable models in medical image analysis: A survey," Med. Image Anal., vol. 1, no. 2, pp. 91-108, 1996.

[20] T. McInerney and D. Terzopoulos, "Topology adaptive deformable surfaces for medical image volume segmentation," IEEE Trans. Med. Imag., vol. 18 , no. 10 , pp. 840-840, Oct. 1999.

[21] S. Osher and R. Fedkiw, Level Set Methods and Dynamic Implicit Surfaces. New York: Springer Verlag, 2003.

[22] S. Osher and N. Paragios, Geometric Level Set Methods in Imaging, Vision, and Graphics. New York: Springer Verlag, 2003.

[23] S. Osher and J. A. Sethian, "Fronts propagation with curvature dependent speed: Algorithms based on Hamilton-Jacobi formulations," J. $C P$, vol. 79, 1988.

[24] N. Paragios and R. Deriche, "Coupled geodesic active regions for image segmentation: A level set approach," ECCV, pp. 224-240, 2000.

[25] M. Rochery, I. H. Jermyn, and J. Zerubia, "Higher order active contours and their application to the detection of line networks in satellite imagery," presented at the IEEE Workshop VLSM, 2003.

[26] M. Rochery, I. H. Jermyn, and J. Zerubia, "Phase field models and higher order active contours," in Proc. ICCV, 2005, vol. 3, pp. III-1096.

[27] M. Rochery, I. H. Jermyn, and J. Zerubia, "Higher order active contours," Int. J. Comput. Vis., vol. 69, no. 1, pp. 27-42, 2006.

[28] G. Russo and P. Smereka, "A remark on computing distance functions," J. CP, pp. 163:51-67, 2000.

[29] S. Kichenassamy, A. Kumar, P. Olver, A. Tannenbaum, and A. Yezzi, "Conformal curvature flows: From phase transitions to active vision," Arch. Rational Mech. Anal., vol. 134, no. 3, pp. 275-301, 1996.

[30] C. Samson, L. Blanc-Féraud, G. Aubert, and J. Zérubia, "A level set model for image classification," Lecture Notes Comput. Sci., vol. 1682, pp. 306-317, 1999.

[31] C. Samson, L. Blanc-Féraud, G. Aubert, and J. Zérubia, "A level set model for image classification," Int. J. Comput. Vis., vol. 40, no. 3, pp. 187-197, 2000.

[32] J. A. Sethian, "A review of recent numerical algorithms for hypersurfaces moving with curvature dependent flows," J. Diff. Geom., vol. 31, pp. 131-161, 1989.
[33] J. A. Sethian, "A fast marching level set method for monotonically advancing fronts," Proc. NAS, 1995.

[34] J. A. Sethian, Level Set Methods and Fast Marching Methods: Evolving interfaces in Computational Geometry, Fluid Mechanics, Computer Vision and Material Science . London, U.K.: Cambridge Univ. Press, 1999.

[35] J. A. Sethian, "Evolution, implementation and application of level set and fast marching methods for advancing fronts," $J$. CP, vol. 169, no. 2, pp. 503-555, 2001.

[36] G. Sundaramoorthi and A. Yezzi, "More-than-topology-preserving flows for active contours and polygons," in Proc. ICCV, 2005, vol. 2, pp. 810-813.

[37] G. Sundaramoorthi and A. Yezzi, "Global regularizing flows with topology preservation for active contours and polygons," IEEE Trans. Image Process., vol. 16, no. 3, pp. 803-812, Mar. 2007.

[38] M. Sussman, P. Smereka, and S. Osher, "A level set approach for computing solutions to incompressible 2-phase flow," $J$. CP, vol. 114, no. 1, pp. 146-159, 1994.

[39] A. Tsai, A. Yezzi, and A. S. Willsky, "Curve evolution implementation of the Mumford-Shah functional for image segmentation, denoising, interpolation, and magnification," IEEE Trans. Image Process., vol. 10, no. 8, pp. 1169-1186, Aug. 2001.

[40] L. A. Vese and T. F. Chan, "A multiphase level set framework for image segmentation using the Mumford and Shah model," Int. J. Comput. Vis., vol. 50, no. 3, pp. 271-293, 2002.

[41] J. Weickert and G. Kühne, Fast Methods for Implicit Active Contour Models. Saarbrücken, Germany: Univ. Saarlandes, 2002.

[42] A. Yezzi, A. Tsai, and A. S. Willsky, "Fully global approach to image segmentation via coupled curve evolution equations," J. VCIR, vol. 13, no. 1-2, pp. 195-1216, 2002.

[43] H.-K. Zhao, T. Chan, B. Merriman, and S. Osher, "A variational level set approach to multiphase motion," J. CP, vol. 127, no. 1, pp. 179-195, 1996.

[44] H.-K. Zhao, S. Osher, B. Merriman, and M. Kang, "Implicit and nonparametric shape reconstruction from unorganized data using a variational level set method," CVIU, vol. 80, no. 3, pp. 295-314, 2000.

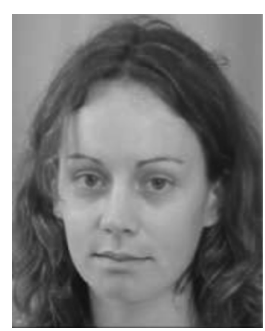

Carole Le Guyader received the French engineering degree (equivalent to M.Sc. degree) in mathematical engineering and the Postgraduate degree in engineering sciences from the National Institute of Applied Sciences (NIAS), Rouen, France, in 2002, and the $\mathrm{Ph} . \mathrm{D}$. degree in applied mathematics from NIAS, Rouen, in 2004 . Her thesis was dedicated to segmentation under geometrical constraints from a theoretical and applied point of view.

She held visiting research positions at the University of California, Los Angeles (Summer 2005 and 2007). She is currently an Assistant Professor at the NIAS, Rennes, France. Her research interests include variational methods in image-related problems, such as segmentation and registration, curve and surface evolution using PDEs, and approximation of surfaces.

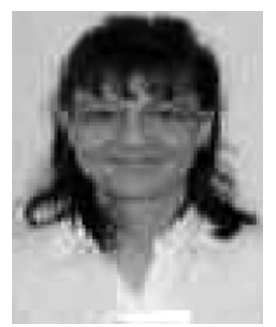

Luminita A. Vese (M'08) received the undergraduate-master degree in mathematics from West University of Timisoara, Romania, in 1993, and the M.S. and Ph.D. degrees in mathematics from the University of Nice, Sophia Antipolis, France, in 1992 and 1997, respectively.

She is an Associate Professor of Mathematics at the University of California, Los Angeles (UCLA). Before joining the faculty at UCLA, she held temporary research and teaching positions at the University of Nice, the University of Paris IX Dauphine, and UCLA. Her research interests include variational methods and partial differential equations, with applications to inverse problems, image analysis, and computer vision. 